\title{
Feature Selection for Global Redundancy Minimization Using Regularized Trees
}

\author{
Shweta Satish Shringarputale ${ }^{1}$, P. R. Rathod ${ }^{2}$ \\ ${ }^{1}$ Department of Computer Science and Engineering Marthawada Shikshan Prasark Mandal's Deogiri Institute of Engineering \& \\ Management Studies, Aurangabad Maharashtra state, India 2015-16 \\ ${ }^{2}$ Associate Professor Department of Computer Science and Engineering Marthawada Shikshan Prasark Mandales Deogiri Institute of \\ Engineering \& Management Studies, Aurangabad Maharashtra state, India 2015-16
}

\begin{abstract}
Feature selection has been a critical exploration subject in data mining, in light of the fact that the genuine data sets frequently have high dimensional elements, for example, the bioinformatics and content mining applications. Numerous current channel feature selection systems rank elements by advancing certain element ranking rules, such that related elements regularly have comparable rankings. These connected components are repetitive and don't give substantial shared data to help data mining. Therefore, when we select a set number of components, we plan to choose the top non-repetitive elements such that the valuable common data can be amplified. In past exploration, Ding et al. perceived this essential issue and proposed the base Redundancy Maximum Relevance Feature Selection (mRMR) model to minimize the repetition between consecutively chose features. In any case, this system utilized the eager hunt, along these lines the worldwide component repetition wasn't considered and the outcomes are not ideal. In this paper, we propose another element selection structure to all inclusive minimize the component excess with boosting the given element ranking scores, which can originate from any regulated or unsupervised strategies. Our new model has no parameter with the goal that it is particularly suitable for functional data mining application. We propose a tree regularization structure, which empowers numerous tree models to perform feature selection efficiently. The key thought of the regularization system is to punish selecting another component for part when its increase (e.g. data increase) is like the elements utilized as a part of past parts. The regularization structure is connected on irregular woods and helped trees here, and can be effectively connected to other tree models. Test studies demonstrate that the regularized trees can choose fantastic element subsets with respect to both solid and frail classifiers. Since tree models can normally manage clear cut and numerical variables, missing qualities, distinctive scales between variables, connections and nonlinearities and so forth. The tree regularization system gives a compelling and effective feature selection answer for many practical problems.
\end{abstract}

Keywords: Feature selection, Feature Ranking, Redundancy Minimization, Regularized Trees

\section{Introduction}

Late quick upgrades in exploration and progressing advancements in data innovation empower us to gather tremendous measures of information. Breaking down these enormous information as turn into a key premise of rivalry, supporting new floods of profitability development, advancement, and purchaser overflow. Numerous information mining and machine learning methodologies have been created to break down and comprehend the experimental information for distinctive applications. Among them, feature selection is one of most imperative procedures, and can upgrade other information mining assignments, for example, order and bunching.

The basic approach that a maximal grouping of contiguous word characters in the content stream constitutes a word. Common content preparing applications standardize the upper forcing so as to case of every word every character to lowercase. Let this change controlled by the character function to LowerCase (char). The mapping is noninsignificant for some Unicode letters. Moreover, if underscores or digits are incorporated among the word characters, then their lower case mapping is the identity function.

As the objective of features selection is to choose a minimized subset of features to speak to information, expect the chose features can give maximal shared data reaction/target variable. There are three sorts of feature selection methods: filter method, wrapper method, and embedded method. The filter methods have low computational expense, yet the chose features frequently can't accomplish great grouping execution. The features chose by wrapper methods more often than not have great execution. $\mathrm{Be}$ that as it may, the wrapper methods use grouping results to choose feature, consequently their computational expense is high and is not suitable for expansive scale applications. The embedded methods fuse feature pursuit and order model into a solitary streamlining issue, and normally is quicker than the wrapper methods and slower than the filter methods.

In spite of the fact that there are a wide range of sorts of feature selection approaches, their component is the same, i.e. every one of them use distinctive approaches to rank features, for example, score capacity, grouping results, weights from the model parameter grid. Subsequently, numerous top positioned features are frequently associated to one another. From measurements perspective, these associated features are excess and more repetitive features may not acquaint additional valuable data with help information mining. Therefore, when select a breaking point number of features, would like to choose the top non-excess features such that the helpful common data can be augmented.

Feature repetition can be measured by the cosine likeness between features. Here utilize worldwide repetition to speak to the aggregate of excess of a feature with every single other 


\section{International Journal of Science and Research (IJSR) \\ ISSN (Online): 2319-7064}

Index Copernicus Value (2013): 6.14 | Impact Factor (2014): 5.611

feature. In past examination, Ding et al. perceived this critical issue and proposed the base Redundancy Maximum Relevance Feature Selection (mRMR) model to minimize the excess between consecutively chose features. To address this issue propose another feature selection system to internationally minimize feature repetition with expanding the positioning scores. The feature positioning consequences of any feature selection method can be utilized as the information of our new system. The proposed model minimizes the worldwide repetition and refines the positioning scores, such that the feature rankings are moved forward. Our new structure can be connected to both unsupervised and managed feature selection methods. Test results on benchmark information sets demonstrate that the proposed global redundancy minimization (GRM) system reliably enhances the feature selection results contrasted with the first methods.

\section{Literature Survey}

Feature selection is to choose significant and instructive elements from the high-dimensional space, and assumes a critical part in numerous investigative and down to earth applications, on the grounds that it can accelerate the learning procedure; enhance the mode speculation ability, and abatement the calculation running time in the genuine applications. The high-dimensional data mining frequently endure the scourge of dimensionality issue, which is regularly brought on by the high-dimensional components and a little size of tests [1]. For instance, in The Cancer Genome Atlas (TCGA) data mining, every specimen has a few ten thousand quality expression measures and a great many single nucleotide polymorphism (SNP) measures, however the tumors or the subtypes of growths are just connected with a little arrangement of qualities or SNPs.

In spite of the fact that, it can utilize the customary measurement diminishment system, i.e. principle component analysis (PCA), linear discriminant analysis (LDA) and so on., to decrease the element size, can't handle the issues where the elements have common implications and can't be numerically joined such as text mining, to select text key words Text feature extraction relies on upon some meaning of which characters are to be dealt with as word characters versus non-word characters [2]. Other than the letters $A-Z$ (in both upper and lower cases variations), word characters might likewise incorporate highlighted letters, digits, the underscore, and by and large all Unicode characters having one of the Unicode "Letter" general classes (uppercase, lowercase, title case, modifier, or other), contingent upon the application. Let the Boolean function is Word (char) decide the status of any given character. For this paper, take the basic approach that a maximal grouping of contiguous word characters in the content stream constitutes a word. Common content preparing applications standardize the upper forcing so as to case of every word every character to lowercase. Let this change controlled by the character function to Lower Case (char). As the objective of features selection is to choose a minimized subset of features to speak to information, expect the chose features can give maximal shared data reaction/target variable.
Ding et al. [3], [4] proposed a covetous methodology (mRMR) to take out the excess features: if one element fi is chosen, different features profoundly corresponded with fi are rejected. On the other hand, these prohibited features may have low worldwide redundancy on the whole, and lead to the non-ideal element selection results.

For instance, there are four features with positioning scores: $10,9.8,9.5,1$ (higher score implies more discriminative), as appeared in Fig. 1. The positioning score of an element is basically controlled by the relationship between's the component and the reaction variable. For the previously stated case, the second and the third features are uncorrelated: the second element clarifies one part of variety accordingly variable and the third components clarify another part of variety. The primary element is corresponded with the second and the third features, and clarifies the greater part of the varieties accordingly variable, in this way most astounding score. In any case, the mix of feature 2 and feature 3 contains more helpful data than feature 1 , subsequently, it is ideal to choose feature 2 and feature 3 . To handle this issue, propose another component selection model for enhancing feature positioning by means of global redundancy minimization.

In literature, discovered the other free work proposed to take care of a comparative issue keeping in mind the end goal to accomplish least redundancy[5]. On the other hand, there is one parameter should be tuned. The model proposed in our work is without parameter. More vital, the relationship framework utilized as a part of may not be sure semiunmistakable, which prompt a non-curved issue, and the worldwide ideal cannot be acquired. Conversely, the positive semi-definiteness of the relationship grid is ensured in our work [6][7][8][9].

In the study, they embrace, rather than ignore, the complexity of the mapping between interconnected imaging measures and interrelated clinical scores; and propose a novel Sparse Multi-tAsk Regression and feaTure selection (SMART) method to jointly analyze all the imaging and clinical data within a single regression model and common subspace[10]. Our research focuses on investigating the relationships between MRI measures and RAVLT memory scores using the Alzheimer's disease Neuro imaging Initiative (ADNI) cohort [11]. Instead of including all possible imaging measures to predict memory performance, the proposed SMART method is designed to select the most prominent imaging features that are able to predict memory performance with improved prediction accuracy. Different from LASSO [12] and other related methods that perform feature selection separately for each individual memory score, the proposed sparse multi-task learning model treats each memory score as a cognition task and selects imaging features that can jointly influence multiple scores/tasks. Here propose to use the combined $\ell 2,1$-norm and $\ell 1$-norm regularizations to select features with high correlations to a subset of memory scores. To demonstrate the effectiveness of the proposed SMART method, apply it to identify relevant MRI markers that can predict multiple RAVLT memory scores. Our empirical results yield not only clearly improved prediction rates in all the test cases, but also a compact set of RAVLT-relevant MRI predictors that are in accordance with prior studies. 


\section{International Journal of Science and Research (IJSR) \\ ISSN (Online): 2319-7064}

Index Copernicus Value (2013): 6.14 | Impact Factor (2014): 5.611

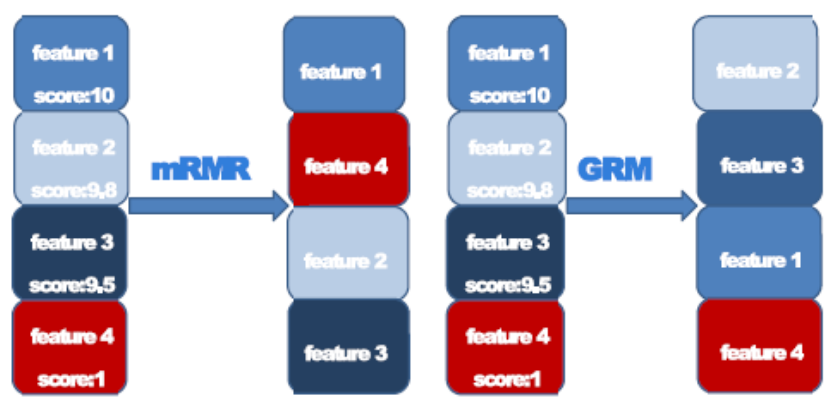

Figure 1: Illustration of mRMR (left) and GRM (right) mechanism. The first feature is correlated with the second and the third features, but the second and the third features are uncorrelated.

Recently sparse regularizations have been applied to classification based feature selection studies. LASSO was shown to efficiently select useful features for a single task. However, in our work, expect to estimate predictive models for several related memory performance scores together, not an individual one. The multi-task feature learning used the $\ell 2,1$-norm regularization to couple feature selection across tasks using a strict assumption - all tasks share a common underlying representation. However, in many cases, the common pattern is shared by many tasks, but not all.

J. Wu and J. M. Rehg, presented CENTRIST (CENsus TRansform hISTogram), a new visual descriptor for recognizing topological places or scene categories. It show that place and scene recognition, especially for indoor environments, require its visual descriptor to possess properties that are different from other vision domains (e.g. object recognition)[13]. CENTRIST satisfy these properties and suits the place and scene recognition task. It is a holistic representation and has strong generalizability for category recognition. CENTRIST mainly encodes the structural properties within an image and suppresses detailed textural information. CENTRIST (CENsus TRansform hISTogram), a visual descriptor that is suitable for recognizing topological places and scene categories. It also show that CENTRIST has several important advantages in comparison to state-of-the-art feature descriptors for place/scene recognition and categorization:

- Superior recognition performance on multiple standard datasets

- Significantly fewer parameters to tune.

- Extremely fast evaluation speed (>50 fps).

- Very easy to implement, with source code publicly available.

Most research in speeding up text mining involves algorithmic improvements to induction algorithms, and yet for many large scale applications, such as classifying or indexing large document repositories, the time spent extracting word features from texts can itself greatly exceed the initial training time [14]. A fast method for text feature extraction that folds together Unicode conversion, forced lowercasing, word boundary detection, and string hash computation. It show empirically that our integer hash features result in classifiers with equivalent statistical performance to those built using string word features, but require far less computation and less memory. The obvious use for faster feature extraction is to process more text per second, run more classifiers per second, or require fewer servers to handle the text processing load. Alternately, where the rate of text per second is limited, the benefit may be to lower the impact on a user's machine, e.g. where text analysis agents operate constantly in the babackground to ,understand ${ }^{\text {ee }}$ the texts the user is reading or writing.

\section{Proposed System}

Regardless of the way that there are an extensive variety of sorts of feature selection approaches, their instrument is the same, i.e. each one of them use different ways to deal with rank features, for instance, score limit, classification results, weights from the model parameter structure. Associated features regularly tend to get similar rankings, in light of the fact that they are viewed as pretty much as imperative for classification. In this way, various top situated features are consistently compared to each other. From estimations viewpoint, these associated features are redundant and more redundant features may not familiarize extra significant information with help data mining. Henceforth, when select a cutoff number of features; here need to pick the top nonredundant features such that the supportive regular information can be increased.

Here utilize global excess to speak to the entirety of repetition of a feature with every single other feature. In past examination, Ding et al. perceived this imperative issue and proposed the minimum Redundancy Maximum Relevance Feature Selection (mRMR) with regularization tree model to minimize the repetition between consecutively chose features. To address this issue, propose another feature selection system to globally minimize feature excess with expanding the ranking scores. The feature ranking after-effects ${ }^{\text {ee }}$ of any feature selection system can be utilized as the information of our new structure.

The proposed model minimizes the global repetition and refines the ranking scores, such that the feature rankings are progressed. Our new structure can be connected to both unsupervised and administered feature selection systems. Trial results on benchmark information sets demonstrate that the proposed global excess minimization (GRM) system reliably enhances the feature selection results contrasted with the first strategies.

In spite of the fact that the managed feature selection routines assume to improve results than the unsupervised ones because of the usages of name data, when the quantity of named information is little and can't speak to the genuine information appropriation, the directed strategies may choose the inaccurate features and prompt the more terrible results than the unsupervised ones. In our examinations, the key point. In this manner, the unsupervised feature selection routines are significant for down to earth applications. There is numerous unsupervised feature selection calculations created. For instance, proposed an unsupervised feature selection calculation by means of iteratively performs follow proportion minimization and $\mathrm{KNN}$ algorithm bunching. Proposed an unsupervised feature selection calculation 


\section{International Journal of Science and Research (IJSR) \\ ISSN (Online): 2319-7064}

Index Copernicus Value (2013): 6.14 | Impact Factor (2014): 5.611

hrough relapse on eigen-vector of Laplacian diagram. In this paper, present another unsupervised feature selection technique, which at the same time considers global change and nearby fluctuation, such that they chose features are both global and neighbourhood discriminative. Joined with our proposed global feature repetition minimization system, the new feature selection strategy beats the related strategies in most trial results.

As the objective of feature selection is to choose a reduced subset of features to speak to information, expect the chose features can give maximal shared data reaction/target variable. On the off chance that feature $i$ and feature $j$ are profoundly corresponded, i.e. the total estimation of relationship coefficient |pij| is vast, it is desirable over hold one feature and overlook the other one for minimization. At the point when the quantity of chose features is settled, the chose features with less repetition can give more common data and show bigger discriminative force. Along these lines, redundant features ought to be disposed of amid the feature selection procedure, such that the ideal and minimal subset of features can be chosen.

For instance, there are four features with ranking scores: 10, 9.8, 9.5, 1 (higher score implies more discriminative), as appeared in Fig. 1. The ranking score of a feature is basically controlled by the relationship between's the feature and the reaction variable. For the previously stated sample, the second and the third features are uncorrelated: the second feature clarifies one part of variety accordingly variable and the third feature clarify another part of variety. The principal feature is connected with the second and the third features, and clarifies the vast majority of the varieties accordingly variable, hence most astounding score. Be that as it may, the blend of feature 2 and feature 3 contains more valuable data than feature 1 , in this way, it is ideal to choose feature 2 and feature 3. On the off chance that select two features from them, the mRMR system will choose the features with scores 10 and 1 . Be that as it may, the perfect result is to choose two features with scores 9.8 and 9.5. Be that as it may, these two features have high repetition with the first feature, and are missed by the insatiable calculation. Therefore, rather than avariciously minimizing the excess ought to minimize the global feature repetition. To handle this issue, in this paper, propose another feature selection model for enhancing feature ranking by means of global excess minimization.

In our work how to choose features for grouping highdimensional information. All the more particularly, it demonstrates to perform consecutive feature selection, which is a standout amongst the most prominent feature selection calculations. It likewise demonstrates to utilize holdout and cross-approval to assess the execution of the chose features.

Here likewise propose a tree regularization system for feature selection in choice trees. The regularization system abstains from selecting another feature for part the information in a tree hub when that feature delivers a comparable increase (e.g. data increase) to features effectively chose, and in this way creates a conservative feature subset.

The regularization system just requires a solitary model to be manufactured, and can be effectively added to an extensive variety of tree-based models which utilize one feature for part information at a hub. Here executed the regularization structure on random forest (RF) and helped trees. Trials exhibit the adequacy and proficiency of the two regularized tree troupes.

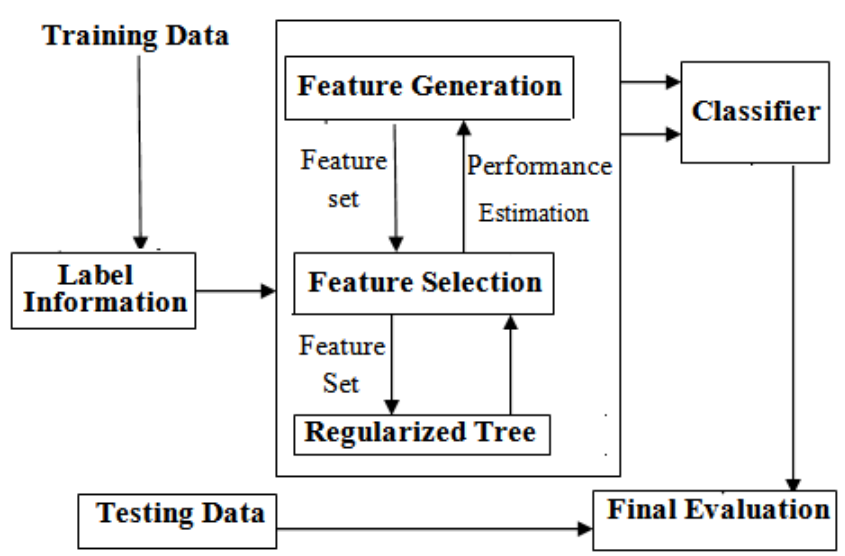

Figure 2: Proposed System Architecture

As tree models normally handle downright and numerical variables, missing qualities, diverse scales between variables, co-operations and nonlinearities and so forth. The tree regularization structure gives a powerful and productive feature selection answer for some reasonable issues.

\section{Conclusion}

In this paper, we have proposed a system for worldwide repetition minimization. The repetition is decreased by applying the GRM system, and classification precision has enhanced essentially for both unsupervised and regulated feature selection calculations. This show the viability of the GRM structure, which minimizes the repetition between chose features, subsequently, the chose features are relied upon to be more conservative and separate. I propose a tree regularization system, which adds feature selection ability to numerous tree models. I connected the regularization structure on arbitrary backwoods and helped trees to produce regularized forms (RRF and RBoost, separately). Trial studies demonstrate that RRF and Rboost produce highquality feature subsets for both solid and feeble classifiers. As tree models are computationally quick and can normally manage straight out and numerical variables, missing qualities, diverse scales (units) between variables, collaborations and nonlinearities and so forth. the tree regularization system gives a powerful and effective feature selection answer for some down to earth issues.

\section{References}

[1] H. Liu and H. Motoda, Feature Selection for Knowledge Discovery and Data Mining. New York, NY, USA: Springer, 1998.

[2] G. Forman and E. Kirshenbaum, "Extremely fast text feature extraction for classification and indexing," in Proc. Int. Conf. Inf. Knowl. Manag., 2008, pp. 12211230 . 
[3] C. Ding and H. Peng, "Minimum redundancy feature selection from microarray gene expression data," J. Bioinformatics Comput. Biol., vol. 3, no. 02, pp. 185205, 2005.

[4] H. Peng, F. Long, and C. Ding, "Feature selection based on mutual information criteria of max-dependency, maxrelevance, and minredundancy,'IEEE Trans. Pattern Anal. Mach. Intell., vol. 27, no. 8,pp. 1226-1238, Aug. 2005.

[5] I. Rodriguez-Lujan, R. Huerta, C. Elkan, and C. S. Cruz,"Quadratic programming feature selection," J. Mach. Learning Res., vol. 11, pp. 1491-1516, 2010.

[6] D. P. Bertsekas. Constrained Optimization and Lagrange Multiplier Methods. Belmont, MA, USA: Athena Scientific, 1996.

[7] M. Hestenes, "Multiplier and gradient methods," J. Optimization Theory Appl., vol. 4, no. 5, pp. 303-320, 1969.

[8] Z. Lin, M. Chen, and Y. Ma, "The augmented lagrange multiplier method for exact recovery of corrupted lowrank matrices," arXiv preprint arXiv:1009.5055, 2010.

[9] M. J. D. Powell, "A method for nonlinear constraints in minimization problems," in Optimization, R. Fletcher, Ed. New York, NY, USA: Academic, 1969.

[10]H. Wang, F. Nie, H. Huang, S. Risacher, C. Ding, A. J. Saykin, L.Shen, and ADNI, "Sparse multi-task regression and feature selection to identify brain imaging predictors for memory performance," in Proc. IEEE Conf. Comput. Vis., 2011, pp. 557-562.

[11] M.W.Weiner, P. S. Aisen, and et al. The alzheimer's disease neuroimaging initiative: progress report and future plans.Alzheimers Dement, 6(3):202-11 e7, 2010.

[12] R. Tibshirani. Regression shrinkage and selection via the LASSO. J. Royal. Statist. Soc B., 58:267-288, 1996.

[13] J. Wu and J. M. Rehg, "CENTRIST: A visual descriptor for scene categorization," IEEE Trans. Pattern Anal. Mach. Intell., vol. 33,no. 8, pp. 1489-1501, Aug. 2011.

[14] G. Forman and E. Kirshenbaum, "Extremely fast text feature extraction for classification and indexing," in Proc. Int. Conf. Inf. Knowl. Manag., 2008, pp. 12211230.

[15]De Wang, Feiping Nie, and Heng Huang, "Feature Selection via Global Redundancy Minimization," IEEE Trans. On Knowledge and Data Engineering, VOL. 27, NO. 10, OCTOBER 2015. 